\title{
Studies of Vertex Tracking with SOI Pixel Sensors for Future Lepton Colliders
}

\author{
Marco Battaglia, ${ }^{\mathrm{a}, \mathrm{b}, \mathrm{c}}$ Devis Contarato, ${ }^{\mathrm{b}}$ Peter Denes, ${ }^{\mathrm{b}}$ \\ Dietrich Liko, ${ }^{\mathrm{d}}$ Serena Mattiazzo, ${ }^{\mathrm{e}}$ Devis Pantano ${ }^{\mathrm{e}}$ \\ ${ }^{a}$ Santa Cruz Institute of Particle Physics, University of California at Santa Cruz, \\ CA 95064, USA \\ ${ }^{\mathrm{b}}$ Lawrence Berkeley National Laboratory, Berkeley, CA 94720, USA \\ ${ }^{\mathrm{c}}$ CERN, CH-1211 Geneva 23, Switzerland \\ ${ }^{\mathrm{d}}$ Institut für Hochenergiephysik, A-1050 Wien, Austria

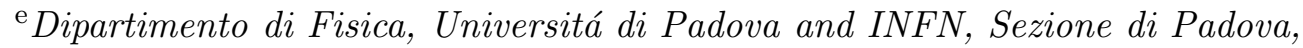 \\ I-35131 Padova, Italy
}

\begin{abstract}
This paper presents a study of vertex tracking with a beam hodoscope consisting of three layers of monolithic pixel sensors in SOI technology on high-resistivity substrate. We study the track extrapolation accuracy, two-track separation and vertex reconstruction accuracy in $\pi^{-} \mathrm{Cu}$ interactions with 150 and $300 \mathrm{GeV} / c$ pions at the CERN SPS. Results are discussed in the context of vertex tracking at future lepton colliders.
\end{abstract}

Key words: Monolithic pixel sensor; SOI technology; Vertex reconstruction.

\section{Introduction}

Vertex tracking is one of the capabilities most crucial to a detector at future lepton colliders and flavour factories. Much of the anticipated physics program at a high energy lepton collider, such as the ILC, CLIC or a muon collider relies on the ability of efficiently discriminating heavy $(t, b, c$ and $\tau)$ from light fermions [1,2]. If the Higgs boson exists at a mass of $\simeq 125 \mathrm{GeV}$, as possibly indicated by the recent preliminary LHC [3, 4] and Tevatron [5] results, the precise determination of its couplings to fermions will be essential to test whether the Brout-Englert-Higgs mechanism of electro-weak symmetry breaking is also responsible for fermion mass generation. Experiments at lower 
energy facilities, such as the Super KEKB [6] and SuperB [7] b-factories, require high resolution vertex tracking for time-dependent measurements, which are crucial to the physics program in heavy flavour physics [8]. Because the momentum of particles from $B$ meson decays at the $\Upsilon(4 \mathrm{~S})$ is low and the sensitivity to short-lived hadrons produced at high energy colliders must extend to most, if not all, of their charged decay products, excellent track extrapolation accuracy at low momentum is of paramount importance. This motivates the development of thin Si sensors. Monolithic pixel sensors, such as CMOS active pixel sensors and DEPFET sensors, have emerged from the R\&D effort for future $e^{+} e^{-}$colliders as the best suited solution for high-resolution vertex tracking with a minimal material budget in a low to moderate radiation environment [9,10,11]. More recently, monolithic pixel sensors on high resistivity substrate have demonstrated superior performance owing to the larger amount of charge collected, ensuring high detection efficiency, and the faster collection time, reducing also the effect of radiation damage [12,13].

Among the technologies offering a CMOS process over a high resistivity sensitive volume, Silicon-On-Insulator (SOI), pioneered a decade ago [14], has been extensively tested in the last six years, within a collaborative R\&D effort developed in partnership with KEK and Lapis Semiconductor Co. Ltd. (formerly OKI Semiconductor). With the mitigation of the back-gating effect using a buried $p$-well (BPW) to protect the in-pixel CMOS circuitry [15], SOI pixel sensor prototypes have demonstrated high detection efficiency and single point resolution at the micron level [16], also after thinning of the handle wafer [17].

In this paper we illustrate the vertex tracking performance of a hodoscope composed of SOI pixel sensors based on the study of inelastic interaction of high energy pions in a beam test (SOIPIX) at the CERN SPS in Fall 2011. We present the results obtained for tracking resolution, two-track separation and vertex resolution, compare our data to simulation and discuss the relation to requirements for future colliders.

\section{Experimental Setup and Data Analysis}

The SOIPIX beam test experiment has been carried out on the SPS beam-line H4 in the CERN North Area with a beam hodoscope made of three layers of "SOImager-2" prototype chips, designed at LBNL and produced in $0.2 \mu \mathrm{m}$ Lapis technology (formerly OKI) on $n$-type SOI wafers with a resistivity of the handle wafer of $\simeq 700 \Omega \cdot \mathrm{cm}$. The sensor sensitive area is a $3.5 \times 3.5 \mathrm{~mm}^{2}$ matrix of $256 \times 256$ pixels arrayed on a $13.75 \mu \mathrm{m}$ pitch, read out through four parallel arrays of 64 columns each [16]. The sensor detection performances for high energy particles and soft X-rays have already been presented in details 
in [16,17,18]. In this setup, a single sensor, thinned to $75 \mu \mathrm{m}$ (singlet), is located upstream from a $\mathrm{Cu}$ target to define the position of impact of the incoming pion. A pair of $260 \mu \mathrm{m}$-thick sensors spaced by $9.4 \mathrm{~mm}$ (doublet) reconstructs the trajectories of the charged particles emerging from the target. This spacing is comparable to that of the two layers of the PXD pixel detector in BELLE-II and to the distance between the first and second layers of a LC vertex tracker. The $3 \mathrm{~mm}$-thick $\mathrm{Cu}$ target is inserted between the detector singlet and the doublet, $15 \mathrm{~mm}$ upstream from the first layer of the SOI pixel doublet. The extrapolation distance from the first doublet layer and the target corresponds to the design distance between the interaction point and Layer0 at SuperB and the first layer of the vertex tracker of an $e^{+} e^{-}$linear collider at $\sqrt{s}$ $=250-500 \mathrm{GeV}$, while for a multi-TeV $e^{+} e^{-}$collider, such as CLIC, the beam induced background requires a stay-clear radius of $\sim 25-30 \mathrm{~mm}$. The use of a thin sensor for the singlet minimises the interaction effects upstream from the target. Data have been collected with $\pi^{-}$s of 150 and $300 \mathrm{GeV} / c$ momentum. Runs with $300 \mathrm{GeV} / c$ pions have also been taken without the target, for alignment and calibration purposes. We operate the sensors at a depletion voltage, $V_{d}$, of $50 \mathrm{~V}$, corresponding to a depleted thickness of $\sim 100 \mu \mathrm{m}$ in the doublet sensors and $\sim 60 \mu \mathrm{m}$ in the thin singlet sensor.

The data acquisition system consists of a custom analog board pigtailed to a commercial FPGA development board, equipped with a Xilinx Virtex-5 FPGA used as control board [19]. The sensor analog outputs are fed to $100 \mathrm{MS} / \mathrm{s}$ 14-bit ADCs through independent analog differential inputs. Digitised data are formatted and transferred to the DAQ computer via a USB-2.0 link at a rate of $25 \mathrm{Mbytes} / \mathrm{s}$. Measurements are performed with the chip clocked at $12.5 \mathrm{MHz}$, corresponding to an $80 \mathrm{~ns}$ read-out time per pixel. The readout is synchronised with the SPS extraction pulse, such that a sequence of 960 frames from each of the three sensors are read-out during the 9.6 s-long SPS spill. Detectors are cooled using forced airflow and the temperature near the sensor surface is in the range $(28 \pm 1)^{\circ} \mathrm{C}$ during data taking. Data sparsification and zero suppression is performed on-line using a custom Root-based [20] program. Sensors are scanned for seed pixels with signal exceeding a preset threshold in noise units. For each seed, the $7 \times 7$ pixel matrix centred around the seed position is selected and stored on file. The pixel pedestal and noise values are updated at the end of each SPS spill, in order to follow possible drifts of their baselines throughout the data taking. Data are stored in Root format and subsequently converted into lcio format [21] for offline analysis.

The data analysis is based on a set of custom processors in the Marlin reconstruction framework [22] to perform cluster centre-of-gravity reconstruction, pattern recognition, track and vertex fitting [23. Clusters are reconstructed applying a double threshold method on the matrix of pixels around a selected candidate cluster seed. Clusters are requested to have a seed pixel with a signal-to-noise ratio, $\mathrm{S} / \mathrm{N}$, of at least 7.0 and the neighbouring pixels with a 
$\mathrm{S} / \mathrm{N}$ in excess of 5.0. Clusters consisting of a single pixel are discarded. The cluster position is computed using the centre of gravity of the measured pulse height. The observed average and most probable signal-to-noise ratio for clusters associated to reconstructed particle tracks in the pixel doublet is 47.4 and 43.0. The most probable value of the signal-to-noise ratio for the seed pixel is 45.1. This can be compared to $45.2 \pm 0.4$ predicted by sensor simulation [17]. The detector planes have been optically surveyed after installation on the beam-line. Their measured positions are used as starting point for the offline alignment procedure with particle tracks, performed using the millipede-2 program [24].

A total of 201000 and 220000 events, having at least one hit per layer in the doublet and only one hit in the singlet, have been recorded in the September 2011 data taking with the target installed and 150 and $300 \mathrm{GeV} / c$ beam, respectively. An additional 20000 calibration events have been collected without the target for alignment.

Simulation of interactions and energy release by charge particles in the detectors is performed using the Geant-4 simulation toolkit [25] with the FTFP_BERT physics list [26], which implements high energy inelastic scattering of hadrons by nuclei using the FRITIOF model [27]. The yield of short- and long-lived particles has been studied using Pythia 6.125 [28]. Charge collection and signal generation in the pixels is simulated using a custom Marlin processor, PixelSim [29], where the pixel $\mathrm{S} / \mathrm{N}$ is tuned to the values obtained in data for each chip. Simulated events are then analysed through the same reconstruction chain as real data.

\subsection{Track Reconstruction}

The efficiency of the SOI sensors is determined by using particle tracks reconstructed on the other two layers and extrapolated to the layer under study, in the events taken without the target.

The Geant-4 + PixelSim simulation predicts a sensor efficiency of $0.998_{-0.012}^{+0.002}$ for the doublet. We estimated the efficiency of the sensors in the doublet in an earlier beam test performed in 2010, when the same SOI sensors were used both in for doublet and the singlet. We use the number of tracks reconstructed from hits on the second layer of the doublet and on the singlet which had an associated hit on the first layer of the doublet and found a value of $0.98_{-0.04}^{+0.02}$ [16]. The single point resolution of the sensors is measured to be $(1.12 \pm 0.03) \mu \mathrm{m}$ for the doublet [16] and $(1.7 \pm 0.50) \mu \mathrm{m}$ for the singlet [17], where simulation predicts $(1.07 \pm 0.04) \mu \mathrm{m}$ and $(1.63 \pm 0.05) \mu \mathrm{m}$, respectively. The difference is due to the lower amount of charge collected, and thus of signal-to-noise ratio, 
in the thin sensor.

Inelastic interaction events are reconstructed from tracks formed with the space points in the two doublet layers. We select events with only one reconstructed hit in the singlet layer and at least one accepted hit in each of the doublet layers to perform the track reconstruction. This pre-selection removes empty events and most of those with more than one primary pion recorded in the same frame.

We reconstruct the trajectory of the incoming pion by projecting the position of the hit reconstructed in the thin singlet. Since the r.m.s. divergence of the beam is $1.5 \times 10^{-4} \mathrm{rad}$ in the vertical and $7.5 \times 10^{-4} \mathrm{rad}$ in the horizontal coordinate, as measured in events taken without the target, the uncertainty on the vertical extrapolation of the pion position of impact on the target is $\simeq 3 \mu \mathrm{m}$ r.m.s. Secondary candidate tracks are reconstructed in the doublet by pairing the hits. These are extrapolated to the singlet and their impact parameter w.r.t. the hit recorded on the singlet sensor computed. Its distribution is peaked at the value of the extrapolation resolution for primary hadrons, which have not interacted in the target, while it has larger values for secondary particles produced in interactions in the target, as well as for primary pions experiencing large scattering, as shown in Figure 1. We resolve

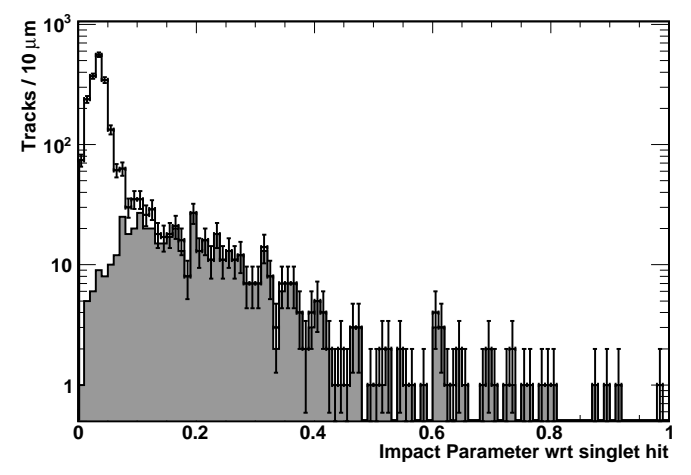

Fig. 1. Impact parameter resolution of tracks reconstructed in the SOI doublet and extrapolated to the singlet hit (in $\mathrm{mm}$ ). The distribution of tracks from inelastic events are shown by the filled histogram.

the combinatorial in the pairing of the doublet hits by choosing the hit pair minimising the impact parameter, provided that the longitudinal position of the intercept of the corresponding track with the estimated primary hadron track is contained in the fiducial region between the singlet layer and the first doublet layer. In simulation, this strategy correctly assigns the hit pairing for 0.96 of the particle tracks emerging from an interaction and contained within the geometrical acceptance of the detector. After this first pass, a vertex is reconstructed for events with two or more tracks. Then, candidate tracks are formed by pairing the unassociated hits in the doublet and tested for compatibility with the interaction vertex by computing their impact parameter w.r.t. 
its position. Candidate tracks with impact parameter smaller than $40 \mu \mathrm{m}$ are kept. Again, the combinatorial is solved by using the hit pair minimising this impact parameter.

\subsection{Vertex Reconstruction}

The interaction vertex for inelastic interaction events is reconstructed with the candidate tracks fitted in the doublet. Several algorithms have been designed to reconstruct the interaction vertex from the trajectories of charged particles. They are typically implemented in the context of collider experiments with a homogeneous magnetic field using a helix track model. Due to the geometry of the beam test setup and the lack of magnetic field, these implementations have numerical problems in reconstructing the interaction vertex for our experimental setup. In absence of a magnetic field, the track model is a simple straight line with four parameters. While the track model is simple, a specific implementation of the vertex fit procedure is required. Due to the low multiplicity in the interaction events, the vertex position $\left(x_{v}, y_{v}, z_{v}\right)$ and the track parameters $p_{i}=\left(x_{i}^{\prime}, y_{i}^{\prime}\right)=\left((d x / d z)_{i},(d y / d z)_{i}\right)$ for particle $i$ can be simply derived from the minimisation of the sum of $\chi_{i}^{2}$ contributions of the trajectory to a global fit. The measurement of the trajectory for particle $i$ on detector $j$ at position $z^{j}$ is given by its Cartesian coordinates $\left(x_{i}^{j}, y_{i}^{j}\right)$ and the corresponding reconstruction errors $\sigma_{x}$ and $\sigma_{y}$. The $\chi_{i}^{2}$ contribution of particle $i$ is then defined as

$$
\chi_{i}^{2}=\sum_{j} \frac{\left(x_{i}^{j}-\left(x_{v}+x_{i}^{\prime}\left(z^{j}-z_{v}\right)\right)\right)^{2}}{\sigma_{x}^{2}}+\frac{\left(y_{i}^{j}-\left(y_{v}+y_{i}^{\prime}\left(z^{j}-z_{v}\right)\right)\right)^{2}}{\sigma_{y}^{2}} .
$$

Monte Carlo simulation studies show that this approach avoids the numerical shortcomings of other implementations of vertex fits. The choice of an adequate track model is essential to reach high efficiency and accuracy in the fitting procedure. In this implementation a global fit has been used for simplicity, but the straight line track model could also be implemented in more complex vertex reconstruction programs. The vertex fit is performed iteratively. If the vertex $\chi^{2}$ probability is smaller than $10^{-4}$, the track giving the largest $\chi^{2}$ contribution is removed and the vertex recomputed. The hits used for reconstructing these tracks are not further used. A reconstructed event is shown in Figure 2. Using fully simulated and reconstructed events, we estimate the vertex reconstruction efficiency for inelastic interaction events with at least two charged particles within the detector acceptance to be $0.94 \pm 0.05$. Further, the reconstructed multiplicity of tracks associated to the vertex agrees with the generated charged multiplicity in the detector acceptance within \pm 0.04 . 


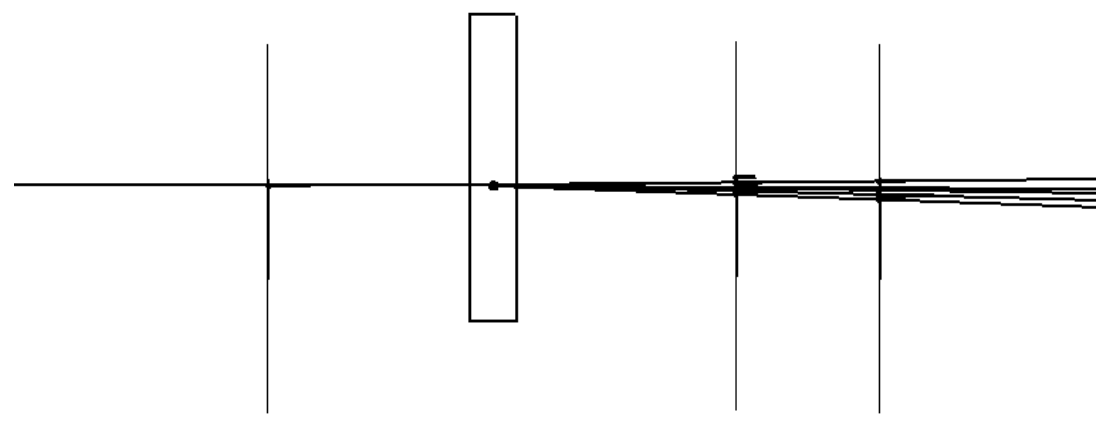

Fig. 2. A $300 \mathrm{GeV} / c \pi^{-}$inelastic interaction event with five charged reconstructed particles. The beam comes from the left and the pion position is first determined in the singlet layer, shown on the left. After the interaction in the target, in the centre of the picture, charged particle tracks are reconstructed on the two doublet layers shown on the right.

\section{Results}

The number of candidate inelastic interaction events, reconstructed in data by the procedure discussed above, is 1105 and 2118 for 150 and $300 \mathrm{GeV} / c$ pions, respectively, corresponding to $0.0055 \pm 0.0002$ and $0.0096 \pm 0.0006$ of the events preselected. The observed fractions agree quite well with simulation, which predicts $0.0065 \pm 0.0003$ and $0.0101 \pm 0.0003$, confirming the efficiency indicated by simulation. For the subsequent analysis we apply additional quality cuts on the reconstructed events requiring the vertex fit $\chi^{2}$ probability to exceed $10^{-4}$ and the uncertainty on the vertex longitudinal position to be smaller than $1.5 \mathrm{~mm}$. This yields a total of 1048 selected interaction events at $150 \mathrm{GeV} / \mathrm{c}$ and 1862 at $300 \mathrm{GeV} / c$.

\subsection{Tracking Resolution}

The track extrapolation accuracy at the position of the $\mathrm{Cu}$ target, located $15 \mathrm{~mm}$ upstream from the first layer of the SOI pixel doublet is $3.7 \mu \mathrm{m}$ for $300 \mathrm{GeV} / c$ and $8.9 \mu \mathrm{m}$ for $1 \mathrm{GeV} / c$ particles according to simulation, as shown in Figure 3. These values are quite comparable to those expected for the vertex tracker proposed for a linear collider, i.e. $\simeq 2 \mu \mathrm{m} \oplus \frac{10 \mu \mathrm{mGeV}^{-1}}{p_{t} \sin \theta}$, where $\theta$ is the track polar angle, for $\sqrt{s}=250-500 \mathrm{GeV}$ with innermost pixel layer at $\simeq 15 \mathrm{~mm}$ radius, and $\simeq 4 \mu \mathrm{m} \oplus \frac{21 \mu \mathrm{m} / \mathrm{GeV}^{-1}}{p_{t} \sin \theta}$, for $\sqrt{s}=3 \mathrm{TeV}$ with innermost layer at $\simeq 30 \mathrm{~mm}$ radius. The impact parameter of tracks w.r.t. the reconstructed vertex is computed for inelastic interaction events with three or more tracks associated to the vertex. Tracks are iteratively removed from the vertex and its position recomputed to avoid the bias due to the contribution 

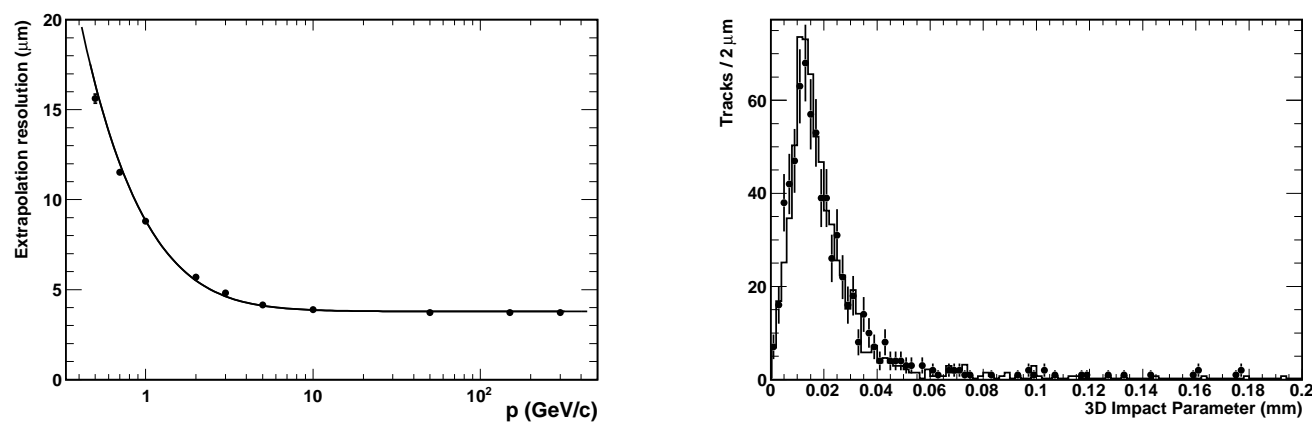

Fig. 3. Track extrapolation resolution on the target using the SOI doublet. Left: the simulation result is shown by the dots with error bars while the fitted curve is $3.7 \mu \mathrm{m} \oplus \frac{8.0 \mu \mathrm{mGeV}^{-1}}{p}$. Right: impact parameter of tracks w.r.t. the interaction vertex. Tracks are removed from the vertex before the impact parameter is computed. Points with error bars represent the data and the line the simulation.

of the track under study. The distance between the track and the interaction vertex is computed at the position of closest approach. The most probable value of the impact parameter resolution is $12.7 \mu \mathrm{m}$, which is the convolution of the track extrapolation resolution, the vertex position resolution $(\simeq 9.0 \mu \mathrm{m}$, see section 3.4) and the multiple scattering in the target. Data and simulation agree well, as shown in Figure 3 .

\subsection{Two-track Separation}

Two-track separation is an important figure of merit for vertex tracking and the optimisation of the pixel sensor geometry. In fact, a given target value of the single point resolution can be achieved with different pixel sizes, $P$, depending on the $\mathrm{S} / \mathrm{N}$ ratio and the charge spread on neighbouring pixels. In the case of the sensor used here, with $P=13.75 \mu \mathrm{m}$, a seed most probable $\mathrm{S} / \mathrm{N}$ value of 45 and $\simeq 0.35$ of the total cluster charge collected on the surrounding pixels, we obtain a $1.1 \mu \mathrm{m}$ resolution for isolated particle tracks. The same resolution could be achieved also with a pitch $P=25 \mu \mathrm{m}$, for a $\mathrm{S} / \mathrm{N} \sim 70$. On the other hand, two-track separation, which scales $\propto P$, and occupancy, which scales $\propto P^{2}$, put emphasis on small pixel size and limited charge spread to minimise cluster merging. In inelastic interaction events we observe a hit density on the first doublet layer of 2.5 hits $\mathrm{mm}^{-2}$. The two-track separation is studied on these events by computing the distance between the two closest reconstructed hits on the two layers of the doublet. Due to the large incoming pion momentum, the interaction products are highly boosted in the forward direction, closely resembling a highly collimated jet at collider experiments. When they are detected on the first layer of the doublet, the minimum hit distance has a most probable value of $70 \mu \mathrm{m}$. In the core of simulated hadronic jets it is $\sim 400 \mu \mathrm{m}$ in $e^{+} e^{-} \rightarrow H^{0} Z^{0} \rightarrow b \bar{b} q \bar{q}$ events at $\sqrt{s}=500 \mathrm{GeV}$ and 

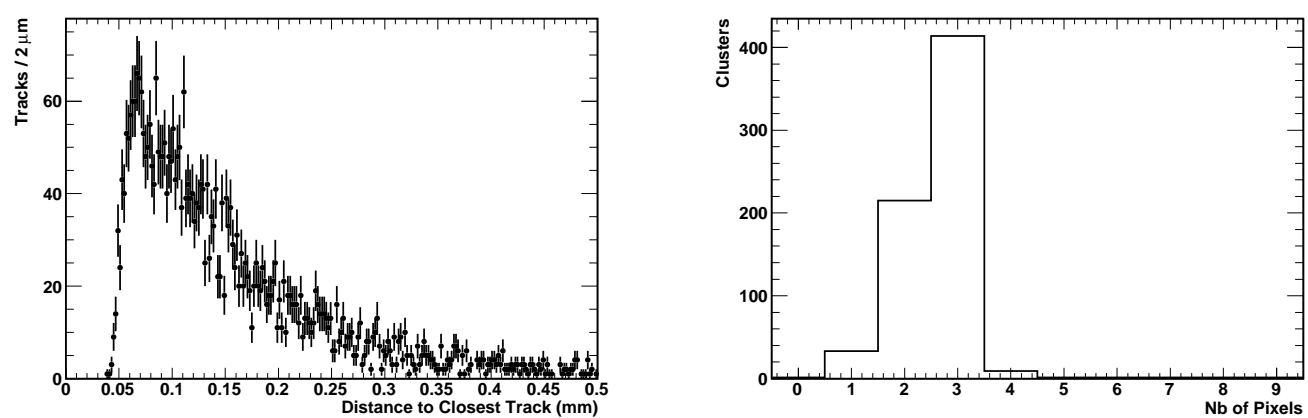

Fig. 4. Two track separation characterisation. Left: minimum distance between a hit associated to a reconstructed track and the closest hit on the first layer of the SOI doublet. Right: number of pixels along rows in clusters associated to tracks.

$\sim 240 \mu \mathrm{m}$ in $e^{+} e^{-} \rightarrow H^{0} A^{0} \rightarrow b \bar{b} b \bar{b}$ events at $\sqrt{s}=3 \mathrm{TeV}$. In our data, we observe a distinct cut-off of the distribution at a minimum hit distance value of $\simeq 50 \mu \mathrm{m}$, corresponding to the smallest two-track separation resolved in the detector. This is comparable to the average size of clusters associated to reconstructed tracks along rows and columns, which is $2.67 \pm 0.6$ pixels, and to its most probable value, 3 pixels corresponding to $42 \mu \mathrm{m}$ (see Figure 4 ). Below this distance, the charge deposited on the pixels by distinct tracks is merged into a single cluster hit. We study the hit merging by selecting events with a
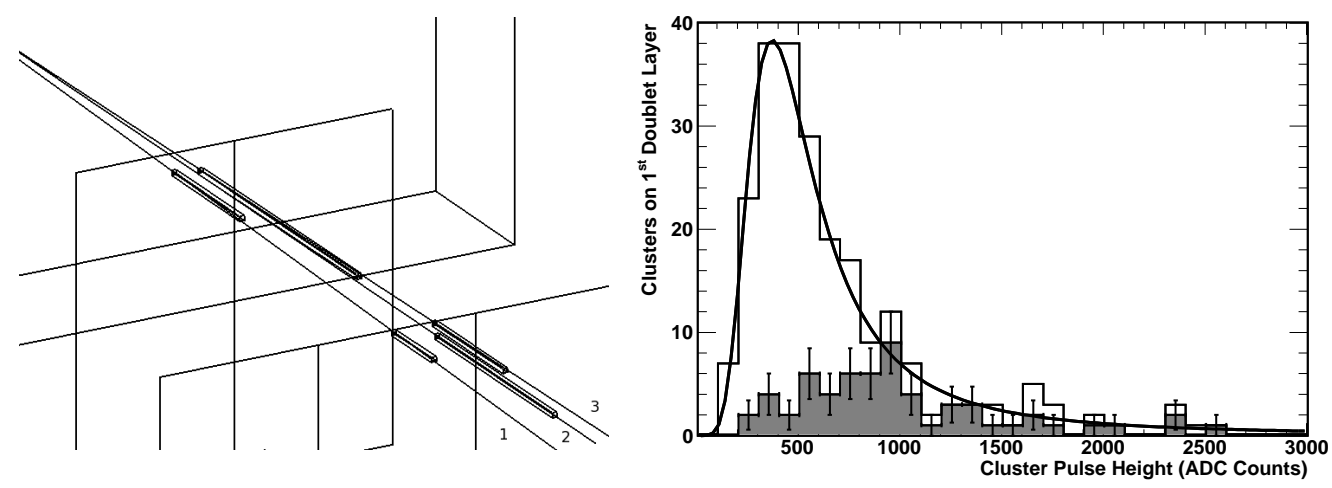

Fig. 5. Two track separation and hit merging. Left: display of event with two tracks (labelled 2 and 3) giving a merged single hit with cluster pulse height of 1200 ADC counts on the first layer of the SOI doublet. Right: Pulse height for clusters on the first layer of the doublet in events with a pseudo-track made with an unassociated hit in the second layer (unfilled histogram). The grey filled histogram shows the pulse height distribution for clusters which are within $50 \mu \mathrm{m}$ from the pseudo-track extrapolation and are already associated to another track. The continuous line shows the fit of a Landau function.

reconstructed interaction vertex and at least one hit in the second SOI doublet layer which is not associated to a track. In these events, a "pseudo-track" is built by fitting a straight line from the unassociated hit to the reconstructed vertex. This pseudo-track is extrapolated to the first layer of the doublet and 
the hit closest to the extrapolation point is selected. Figure 5 shows one of such events and the cluster pulse height of all these hits with highlighted those having a distance from the extrapolated point smaller than $50 \mu \mathrm{m}$, i.e. three and half times the pixel pitch, and which are already associated to another reconstructed track. This category is characterised by a most probable value of the cluster charge of $(772 \pm 80)$ ADC counts, to be compared to $395 \pm 15$ for the distribution of all the selected hits, i.e. larger by a factor of $1.95 \pm 0.02$. This shows that tracks impacting on the detector at a distance closer than 3-4 pixels produce merged hit clusters, characterised by a most probable value

of the collected charge which is approximately double compared to that of isolated clusters. This characteristic can be used, in principle, for identifying them.

It is useful to compare these results to the anticipated track density and twotrack distance at high energy lepton colliders. These are driven by tracks in collimated hadronic jets and the amount of machine-induced backgrounds, mostly incoherent pairs and $\gamma \gamma \rightarrow$ hadrons events, integrated in a read-out cycle. In the process $e^{+} e^{-} \rightarrow H^{0} A^{0} \rightarrow b \bar{b} b \bar{b}$ at $\sqrt{s}=3 \mathrm{TeV}$, the fraction of particles from a $b$ hadron decays reaching the first layer of the Vertex Tracker, at a radius of $30 \mathrm{~mm}$, and within less than $50 \mu \mathrm{m}$ from another charged particle track is 0.03 . The probability to have a charged particle from beam-induced background closer than $50 \mu \mathrm{m}$ is 0.02 assuming a detector integration time of $25 \mathrm{~ns}$, corresponding to 50 bunch crossings.

\subsection{Interaction Multiplicity}

The charged multiplicity of inelastic interactions is measured from the number of particles associated to a reconstructed vertex, as discussed above. In order to compare with existing data, we include one-prong interaction events. These are events having a single reconstructed track in the SOI doublet, which has a longitudinal intercept with the primary pion trajectory in the range $16<z<$ $23 \mathrm{~mm}$, i.e. compatible with the target position, and an impact parameter w.r.t. the position of the hit in the SOI singlet larger than $100 \mu \mathrm{m}$. We obtain a multiplicity of $2.16 \pm 0.18$ and $2.57 \pm 0.22$ for 150 and $300 \mathrm{GeV} / c \pi^{-}$beam, respectively (see Figure 6). The measured multiplicity scales logarithmically with the beam energy, as expected [30]. We compare the multiplicity measured in our data as a function of $s$ to a fit to published data scaled to the rapidity range covered by our detector. Following [31], we use a function of the form $<n>=a+b \ln \left(s / s_{0}\right)$, where the parameters $a$ and $b$ are fitted on results reported for $\pi \mathrm{Cu}$ collisions at beam energies from 50 to $200 \mathrm{GeV} / c$ [32]. We get $a=2.32, b=0.84$ for $s_{0}=392 \mathrm{GeV}^{2}$. Our results agree with this curve, as shown in Figure 7. 

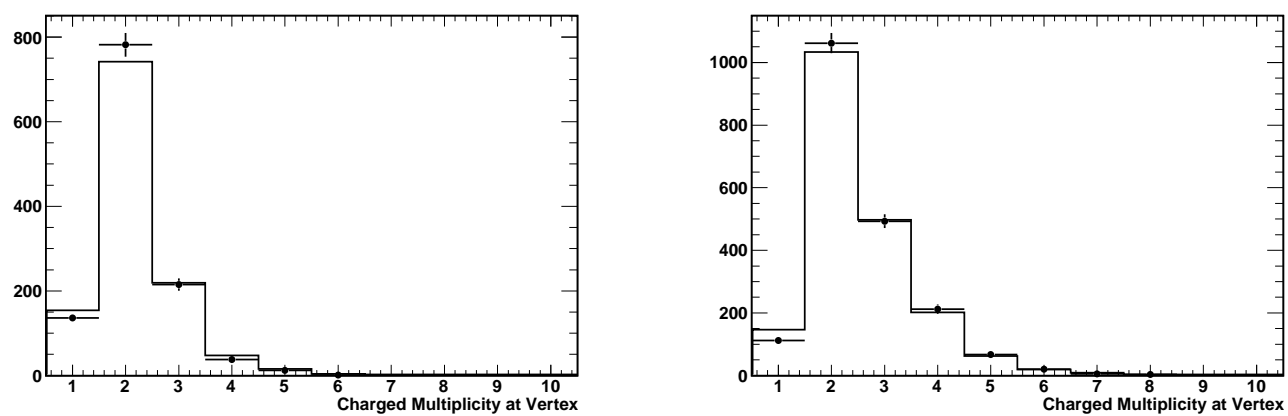

Fig. 6. Reconstructed charged multiplicity of inelastic interaction events for 150 (left) and $300 \mathrm{GeV} / c \pi^{-}$s (right). Data are shown by points with error bars and simulated and reconstructed events by the continuous line.

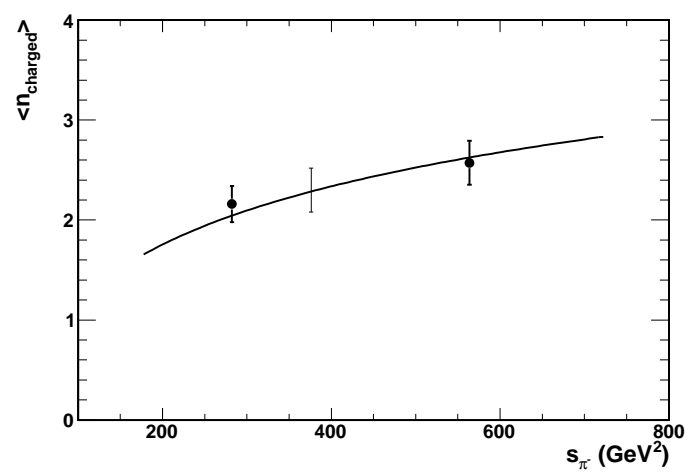

Fig. 7. Average charged multiplicity of inelastic interaction events as a function of $s$. Data are shown by points with error bars and the continuous line represents the fit to earlier data rescaled to the same rapidity interval. The error bar associated to the line represents the uncertainty of the points used for the fit.

\subsection{Vertex Resolution}

The resolution on the vertex position reconstruction in the plane transverse to the beam is obtained by comparing the reconstructed vertex position to that of the incoming pion detected in the thin sensor upstream from the target. We measure a Gaussian width of $(8.9 \pm 0.2) \mu \mathrm{m}$. By subtracting in quadrature the incident pion extrapolation resolution of $3 \mu \mathrm{m}$ (see Section 2.1), we obtain $(8.4 \pm 0.2) \mu \mathrm{m}$, which accounts for the vertex resolution, the multiple scattering of the incident pion in the target before the interaction and that of the emerging interaction products. The estimated average uncertainty from the vertex fit error is $6.2 \mu \mathrm{m}$ and the difference between the generated and reconstructed position on simulated events has a Gaussian width of $6.5 \mu \mathrm{m}$.

The resolution on the longitudinal vertex position is extracted first from the distribution of the longitudinal position of the vertex reconstructed in data and simulation, shown in Figure 8. Since the target has sharp edges, in ab- 
sence of resolution effects this distribution is a box function. Resolution effects introduce a smearing of the box edges, which can be observed in both data and the simulated and reconstructed interaction events. These vary depending on the momentum and multiplicity of the detected particles emerging from the interaction and on the longitudinal position of the interaction in the target. We perform a multi-parameter $\chi^{2}$ fit to the distribution of the reconstructed vertex longitudinal position. As a consistency test, we obtain a measurement of the target thickness of $(3.15 \pm 0.10) \mathrm{mm}$ in data and $(3.07 \pm 0.05) \mathrm{mm}$ in simulation, where a $3.00 \mathrm{~mm}$ thickness was generated. The average longitudinal vertex position resolution from the fit at $300 \mathrm{GeV} / c$ is $(503 \pm 60) \mu \mathrm{m}$ and $(532 \pm 39) \mu \mathrm{m}$, again in data and simulation respectively. It becomes $(615 \pm 83) \mu \mathrm{m}$ and $(590 \pm 35) \mu \mathrm{m}$ for data and simulation, respectively, at $150 \mathrm{GeV} / c$ where the multiplicity is lower and the multiple scattering is more important.

We observe that the distribution of the vertex position away from the target is asymmetric, as we reconstruct more vertices downstream from the target than upstream. Since resolution and pattern recognition failures should give spurious vertices on either sides of the target position with equal probability, we interpret this as due to secondary vertices of long-lived particles produced in the primary interaction and decaying away from the target. Simulation shows a similar effect, mostly due to $K_{S}^{0} \rightarrow \pi^{+} \pi^{-}$decays, at a comparable rate as in our data.

In data, the vertex fit error gives a most probable value of $(335 \pm 7) \mu \mathrm{m}$ and an average value of $(490 \pm 6) \mu \mathrm{m}$, for the longitudinal vertex position resolution at $300 \mathrm{GeV} / c$. The difference in the position of the generated and reconstructed vertices in simulated events has a Gaussian width of $(290 \pm 10) \mu \mathrm{m}$ and nonGaussian tails extending further, with the contribution of multiple scattering in the target to the Gaussian resolution estimated to be $60 \mu \mathrm{m}$, at $300 \mathrm{GeV} / c$. Given the small angular coverage of our hodoscope and the large boost of

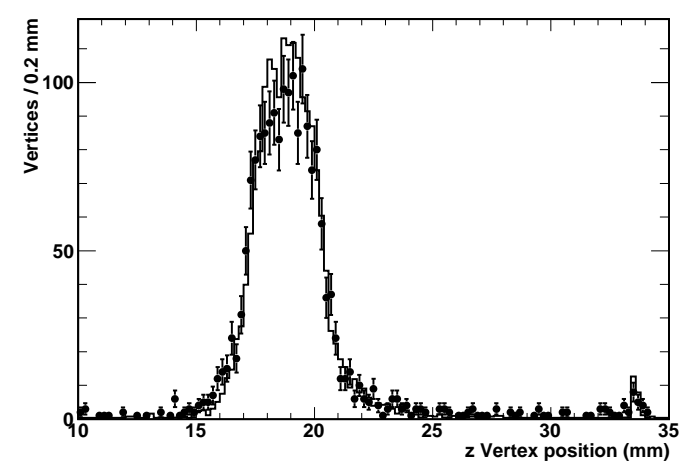

Fig. 8. Distribution of the longitudinal position of fitted vertices in inelastic interactions of $300 \mathrm{GeV} / c \pi^{-}$s. The points with error bars show the distribution for data and the continuous line for simulated and reconstructed events. 
the interaction products, the resolution on the longitudinal vertex position is driven by the small opening angle of the detected interaction products. In $500 \mathrm{GeV} e^{+} e^{-}$collisions, simulated vertices from $B$ mesons with energies in the range $100-150 \mathrm{GeV}$, re-weighted by the associated track multiplicity to match that of the reconstructed vertices in our data, give a resolution of $(170 \pm 20) \mu \mathrm{m}$ along the heavy meson line of flight [23]. On the other hand, at a multi-TeV collider the typical resolution for high energy jets is larger by a factor of two. Decay vertices in $e^{+} e^{-} \rightarrow H^{0} A^{0} \rightarrow b \bar{b} b \bar{b}$ events at $\sqrt{s}=$ $3 \mathrm{TeV}$, where the $B$ mesons have energies in the range $200-700 \mathrm{GeV}$, have been reconstructed using the ZVTOP topological vertex algorithm [33]. The reconstructed secondary decay vertex has an average charged multiplicity of 2.36 and the most probable and average values of the vertex position resolution along the jet axis are $(330 \pm 5) \mu \mathrm{m}$ and $(530 \pm 3) \mu \mathrm{m}$, respectively. These values are very close to those obtained in the present study.

\section{Conclusions}

Tracking and vertexing performance of a small beam hodoscope made of SOI pixel sensors have been determined in a beam test with 150 and $300 \mathrm{GeV} / c$ $\pi^{-} \mathrm{s}$ at the CERN SPS. The charged multiplicity of inelastic $\pi-\mathrm{Cu}$ interactions has been determined to be $2.16 \pm 0.18$ at $150 \mathrm{GeV} / c$ and $2.57 \pm 0.22$ at $300 \mathrm{GeV} / c$, which agrees with earlier data and logarithmic scaling with the beam energy. The two-track separation of the sensor is $\simeq 50 \mu \mathrm{m}$, i.e. four pixels, which agrees with the observed pixel multiplicity along columns and rows in reconstructed clusters. Merged hits can be identified by the large collected charge in the cluster. The vertex resolution is estimated to be $8.4 \mu \mathrm{m}$ transverse and $\simeq 350-500 \mu \mathrm{m}$ longitudinal. The results obtained are representative of the experimental conditions and reconstruction performance expected in highly boosted hadronic jets at TeV-class lepton colliders and demonstrate that pixels sensors with $\sim 15 \mu \mathrm{m}$ pitch on high-resistivity Si are extremely well suited for these applications.

\section{Acknowledgements}

This work was supported by the Director, Office of Science, of the U.S. Department of Energy under Contract No.DE-AC02-05CH11231 and by INFN, Italy. We are grateful to Y. Arai for his effective collaboration in the SOIPIX activities, D. Bisello for his support and P. Giubilato for contributions to the data acquisition system. We are indebted to I. Efthymiopoulos and M. Jeckel for support on the SPS beam-line and to A. Behrens and E. Lacroix for per- 
forming the detector alignment. We also thank the CERN IT department for computing support.

\section{References}

[1] S. Xella Hansen [LCFI Collaboration], Nucl. Instrum. and Meth. A 501 (2003) 106.

[2] M. Battaglia, Nucl. Instrum. and Meth. A 650 (2011) 55.

[3] G. Aad et al. [ATLAS Collaboration], Phys. Lett. B 710 (2012) 49.

[4] S. Chatrchyan et al. [CMS Collaboration], Phys. Lett. B 710 (2012) 26.

[5] The TEVNPH Working Group for the CDF and D0 Collaborations, arXiv:1203.3774 [hep-ex].

[6] Z. Dolezal [Belle II Collaboration], PoS HQL 2010 (2010) 079.

[7] M. Bona et al. [SuperB Collaboration], Report INFN/AE-07/2, arXiv:0709.0451 [hep-ex].

[8] F. Forti et al., Nucl. Instrum. and Meth. A 636 (2011) S168.

[9] A. Besson et al., Nucl. Instrum. and Meth. A 568 (2006) 233.

[10] C. Marinas and M. Vos, Nucl. Instrum. and Meth. A 650 (2011) 59.

[11] G. Rizzo et al., Nucl. Instrum. and Meth. A 650 (2011) 169.

[12] A. Dorokhov et al., Nucl. Instrum. and Meth. A 650 (2011) 174.

[13] M. Deveaux et al., JINST 6 (2011) C02004.

[14] J. Marczewski et al., IEEE Trans. Nucl. Sci. 51 (2004) 1025.

[15] Y. Arai et al., Nucl. Instrum. and Meth. A 623 (2010) 197.

[16] M. Battaglia et al., Nucl. Instrum. and Meth. A, 654 (2011) 258.

[17] M. Battaglia et al., Nucl. Instrum. and Meth. A 676 (2012) 51.

[18] M. Battaglia et al., Nucl. Instrum. and Meth. A, 674 (2012) 50.

[19] M. Battaglia et al., Nucl. Instrum. and Meth. A 611 (2009) 105.

[20] R. Brun and F. Rademakers, Nucl. Instrum. and Meth. A 389 (1997) 81.

[21] F. Gaede et al., in Proc. of Interlaken 2004, Computing in high energy physics and nuclear physics, Report CERN 2005-002, 471.

[22] F. Gaede, Nucl. Instrum. and Meth. A 559 (2006) 177. 
[23] M. Battaglia et al., Nucl. Instrum. and Meth. A 593 (2008) 292.

[24] V. Blobel, Nucl. Instrum. and Meth. A 566 (2006) 5.

[25] S. Agostinelli et al., Nucl. Instrum. and Meth. A 506 (2003) 250.

[26] J. Apostolakis et al., J. Phys. Conf. Series 160 (2009) 012073.

[27] B. Andersson, G. Gustafson and H. Pi, Z. Phys. C 57 (1993) 485.

[28] T. Sjöstrand et al., Comp. Phys. Commun. 135 (2001) 238.

[29] M. Battaglia, Nucl. Instrum. and Meth. A 572 (2007) 274.

[30] Z. Koba, H.B. Nielsen and P. Olesen, Nucl. Phys. B 40 (1972) 317.

[31] A. Firestone et al., Phys. Rev. D 14 (1976) 2902.

[32] J.E. Elias et al., Phys. Rev. D 22 (1980) 13.

[33] D. Bailey et al. [LCFI Collaboration], Nucl. Instrum. and Meth. A 610 (2009) 573. 